\title{
A design and implementation of Pulse-Measure instrument based on Microcontroller
}

\author{
Zhu Siqing ${ }^{1}$, Zhang Ying ${ }^{1, a}$, Liu Yanming ${ }^{1}$, Hang $\mathrm{Yu}^{1}$, Sun Kexue $\mathrm{K}^{1,2, \mathrm{~b}}$ and \\ Cheng Xiefeng ${ }^{1,2, c}$
}

${ }^{1}$ School of Electronic Science and Engineering, Nanjing University of Posts and Telecommunications, Nanjing, 210023, China

${ }^{2}$ Jiangsu Province Engineering Lab of RF integration \& Micropackage, Nanjing, 210023, China

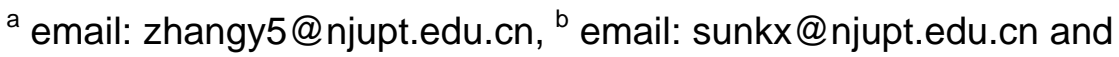

c email: chengxf@njupt.edu.cn

Keywords: Pulse measure; Microcontroller; photoelectric sensor;

\begin{abstract}
Pulse-measure instrument has been widely used in our daily life. How to keeping monitoring pulses has risen to a vital important issue. This design uses infrared photoelectric sensor to measure heart rates by detecting blood which will get thicker when heart beats. The system using the AT89C51 microcontroller as the core to control the whole procedure. A LCD1602 is also comprised which will display the times your heart beats every minute. The upper limit and the lower limit setting by an additional keypad can also be showed on LCD. A buzzer will be alarmed when pulse number is ranging over lower limit and upper limit. This system is very portable and cheap.
\end{abstract}

\section{Introduction}

Observing the pulse signal to check health is usually used in health centers and hospitals. Using human physiological information acquired the basis for clinical diagnosis and treatment has been attached vital importance since ancient time. The pulse wave can present wave form, amplitude, velocity, period and the comprehensive information, which are significant blood characteristics. So measuring pulse and how to detecting the signal have drawn a great attention[1-3].

There are some pulse measurements using pressure sensor. The measuring principle is placing pressure resistance sensor on the radial artery to detect the pulsation. This sensor has the characteristic of strong anti-interference. But the pressure of arterial blood vessel is very light, so the sensor might miss the signal[4,5].

In this paper, photoelectric sensor is used to detect the pulse signal. The pulse signal is a kind of weak signal of low frequency mixed up with lots of noise signal to a great extent, so it must be amplified and filtered to meet the requirements of the collection.

\section{System architecture}

In order to make the measurement ordinary and portable, this design is based on 51 SCM. It uses ST188 reflective photoelectric sensor to collect pulse signal and also includes power button, amplifier-rectifier, liquid-crystal display module, additional keypad and a buzzer, as is shown in the Figure 1. 


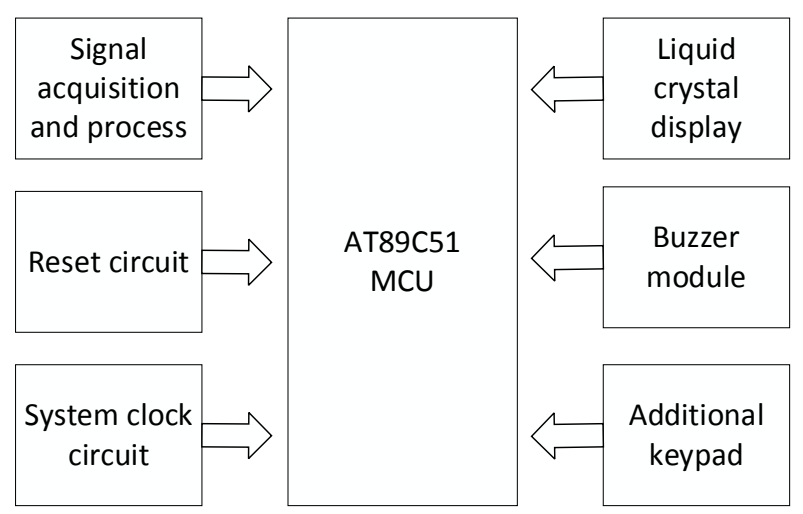

Figure 1. System Architecture

The system contains four buttons which are divided into two parts, one is to reset the MCU, the other's function is setting the upper limit and the lower limit. Placing finger-tip on the sensor can return pulse signal by detecting the change of blood thickness. Then the signal will be transmitted to MCU's I/O port after amplified by LM358. Microcontroller count the pulse time via external interrupt, and then show the number on the LCD. At the meantime, there is also a locking press-button can control the power supply.

\section{Hardware architecture}

This figure details that the hardware system of this design is divided into five parts. Below is the details of them.

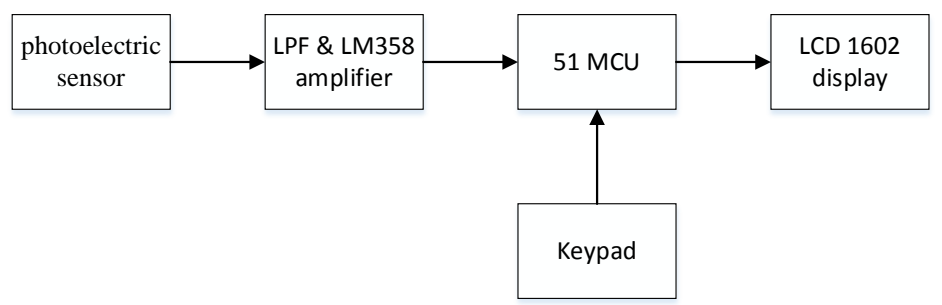

Figure 2. Hardware Architecture

1. Photoelectric sensor, filter and amplifier

Photoelectric sensor: ST188 reflective photoelectric sensor made up by an infrared receiving diode and an infrared emitting diode. This sensor will detect the intensity change of the light through fingertip blood when heart is beating and convert heart beat to electrical signals. When it is used, a current-limiting resistor must be contained.

Filter and amplifier: The pulse signal is very weak, usually in the $\mu \mathrm{V}$ level, along with massive noise interference, so we build up an amplifier and reshaping circuit with LM358 and use three order RC low-frequency pass filter because the frequency of heart beat is in the Hz level.

2. Microcontroller

The AT89C51 is a low-power, high-performance CMOS 8-bit microcomputer with 4Kbytes of Flash programmable and erasable read only memory (PEROM). By combing a versatile 8-bit CPU with Flash on a monolithic chip, the AT89C51 is a powerful microcomputer which provides a highly-flexible and cost-effective solution to many embedded control applications. Below is the oscillator connections circuit of AT89C51. 


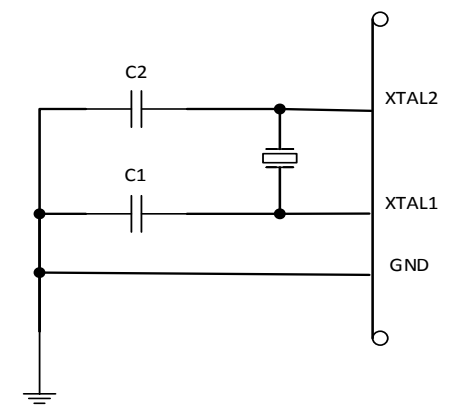

Figure 3. Oscillator Connections

XTAL1: Input to the inverting oscillator amplifier and input to the internal clock operating circuit.

XTAL2: Output from the inverting oscillator amplifier.

C1, C2=20 40pF for crystals, 30 50pF for Ceramic Resonators 。

\section{Software algorithm}

The software and hardware circuit should be designed at the same time, so that which part completed by hardware and which assignment accomplished by software will be confirmed when hardware circuit design is mainly finished. The software system uses the modular architectural design which is similar to the hardware design to get the combination of the system function more convenient. The software has interrupt counting program, additional key procedures, LCD program, etc. as is shown in the Figure 4, 5, 6. Each module can function well as an independent part.

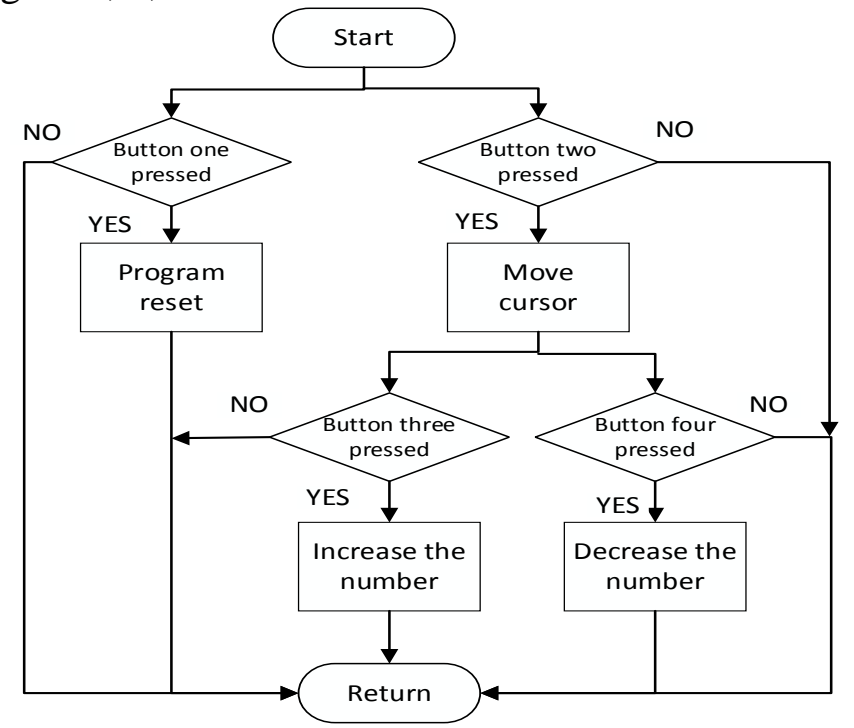

Figure 4. Additional keypad procedure 


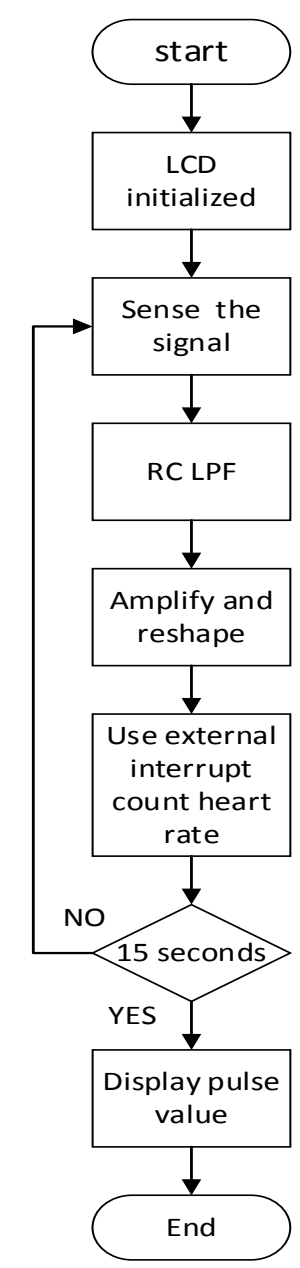

Figure 5. Signal processing and pulse calculation procedure

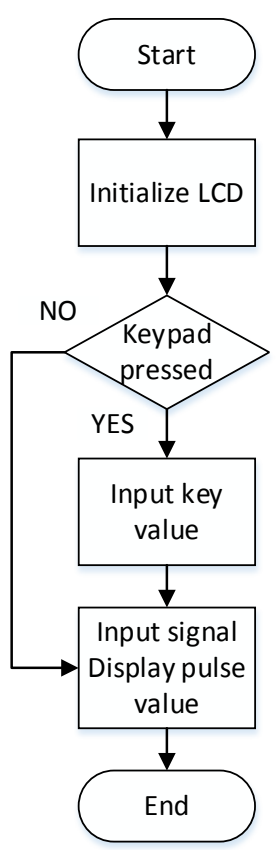

Figure 6. LCD display procedure

\section{Test results}

According to the Table 1, deviations are founded because of the sensor and other devices are not ideal. But the deviation can be tolerated.

Table 1. Test data

\begin{tabular}{|c|c|c|c|c|c|}
\hline $\begin{array}{c}\text { Real data } \\
\text { (per minute) }\end{array}$ & $\begin{array}{c}\text { Test data } \\
\text { one }\end{array}$ & $\begin{array}{c}\text { Test data } \\
\text { two }\end{array}$ & $\begin{array}{c}\text { Test data } \\
\text { three }\end{array}$ & $\begin{array}{c}\text { Test data } \\
\text { four }\end{array}$ & $\begin{array}{c}\text { Test data } \\
\text { five }\end{array}$ \\
\hline 65 & 64 & 64 & 63 & 65 & 63 \\
\hline 72 & 70 & 71 & 69 & 69 & 70 \\
\hline 76 & 76 & 75 & 75 & 74 & 73 \\
\hline 71 & 70 & 70 & 69 & 71 & 70 \\
\hline 75 & 73 & 73 & 75 & 72 & 74 \\
\hline
\end{tabular}

NOTE: The real number is measured by stethoscope

\section{Conclusion}

A photoelectric sensor is used to detect the pulse signal in this paper. The pulse signal is a kind of weak signal of low frequency mixed up with lots of noise signal to a great extent, so it must be amplified and filtered to meet the requirements of the collection. A wireless communication module 
can be added so that pulse data will be uploaded to Internet and the different data can be analyzed by the following data processing. We expect this system will be benefit for the need in their daily life.

\section{Acknowledgement}

This research was financially supported by the National Natural Science Foundation of China (Grant No.61271334), Natural Science Foundation of the Jiangsu Higher Education Institutions of China (Grant No. 15KJD510001), and Natural Science Foundation of Nanjing University of Posts and Telecommunications (Grant No. NY214049).

\section{References}

[1] V.V. Boronoev, O.S. Rinchinov. Some Aspects of A Program Implementation: for A Computer-assisted System for Pulse Diagnosis. Biomedical Engineering. 1996, 30(5): 286-287

[2] X.S. Che, Z.X. Zhang, Y.J. He. The Acquisition and Direct Description of Pulse in Chinese Medicine. ICEMI'2005 Conference Proceedings. 2005,1(6): 49-52

[3] Kennedy, G.; Davis, B. (1992). Electronic Communication Systems (4th ed.). McGraw-Hill International. ISBN 0-07-112672-4, p 509

[4] Dandan Song, Lijuan Jia, Yao Lu. Heart Sounds Monitor and Analysis in Noisy Environments [J]. 2012 International Conference on Systems and Informatics, 2012 1677-1681.

[5] Kexue Sun, Hao Zheng etc. A Design and Implementation for Optical Fibre Vibration Sensor [J]. Applied Mechanics and Materials. Vols.241-244 (2013) : 976-979. 